\title{
Distribution of epiphytic macroalgae on the thalli of their hosts in Cuba
}

\author{
Yander Luis Diez García ${ }^{1}$, Abdiel Jover Capote ${ }^{2,6}$, Ana María Suárez Alfonso², \\ Liliana María Gómez Luna ${ }^{4}$ and Mutue Toyota Fujiii
}

Received: 4 April, 2012. Accepted: 14 October, 2013

\begin{abstract}
We investigated the distribution of epiphytic macroalgae on the thalli of their hosts at eight localities along the southeastern coast of Cuba between June 2010 and March 2011. We divided he epiphytes in two groups according to their distribution on the host: those at the base of the thallus and those on its surface. We determining the dissimilarity between the zones and the species involved. We identified 102 taxa of epiphytic macroalgae. There were significant differences between the two zones. In 31 hosts, the number of epiphytes was higher on the surface of the thallus, whereas the number of epiphytes was higher at the thallus base in 25 hosts, and the epiphytes were equally distributed between the two zones in five hosts $(\mathrm{R}=-0.001, \mathrm{p}=0.398)$. The mean dissimilarity between the two zones, in terms of the species composition of the epiphytic macroalgae, was $96.64 \%$. Hydrolithon farinosum and Polysiphonia atlantica accounted for $43.76 \%$ of the dissimilarity. Among macroalgae, the structure of the thallus seems to be a determinant of their viability as hosts for epiphytes.
\end{abstract}

Key words: Chlorophyta, epiphytism, distribution, Phaeophyceae, Rhodophyta

\section{Introduction}

The structure of intertidal marine communities is determined by a combination of physical factors and biotic interactions (Little \& Kitching 1996; Wernberg \& Connell 2008). The establishment of marine macroalgae involves a number of complex physical interactions, as well as biological, ecological and chemical processes at the microscale, from the release of propagules by reproductive adults to their migration to appropriate substrates, initial adhesion to a surface, permanent attachment and development (Reed 1990a; Brawley \& Johnson 1992; Vreeland \& Epstein 1996). Because macroalgae constitute a key component of coastal communities, they are associated with other organisms and processes and have been central to the debate about the nature and scope of the structure and dynamics of those communities (Foster 1990, 1991; Paine 1991; Underwood 2000).

In the intertidal zone, environmental gradients also occur at smaller scales, such as within the host zone of receptivity, where there is a light intensity gradient between the periphery and the interior of the thallus, and along host fronds, where there is a gradient in biomass, which decreases progressively from the holdfast to the frond tip. The investigation of the distribution of epiphytes at different scales is a potentially productive approach. It is important to understand the patterns of abundance of all sympatric epiphytic species along the various gradients, because interspecific relationships could represent one of the factors that explain their distribution (Longtin et al. 2009).

The texture and structure of the host thalli are frequently exploited by epiphytes, thus increasing habitat complexity (Hacker \& Steneck 1990). The epiphytic macroalgae with a filamentous or branched structure usually present a high degree of structural complexity and might therefore increase the suitability of host algae as habitats for mesoherbivores (Martin-Smith 1993).

In marine communities, the plant substrates that are the most widely colonized by are seagrasses and macroalgae, because they provide the necessary space, shelter and nutrition (Aguilar-Rosas \& Galindo 1990). Macroalgae morphology might be related to the density of the community (Reed 1990b; Ang \& DeWreede 1992). High densities of adults can help reduce desiccation during low tide due to overlapping thalli, as well as reducing water flow, providing shade and microhabitats below, which can affect the structure of the community (Bruno et al. 2003).

The host macroalgae differ considerably in their suitability as substrates for epiphytes. This difference might be

\footnotetext{
${ }^{1}$ Administración Portuaria Santiago de Cuba, Santiago de Cuba, Cuba

${ }^{2}$ Universidad de Oriente, Departamento de Biología, Santiago de Cuba, Cuba

${ }^{3}$ Universidad de La Habana, Centro de Investigaciones Marinas, Ciudad Habana, Cuba

${ }^{4}$ Universidad de Oriente, Centro Nacional de Electromagnetismo Aplicado, Laboratorio de Ecotoxicología, Santiago de Cuba, Cuba

${ }^{5}$ Instituto de Botânica, Seção de Ficologia, São Paulo, SP, Brazil

${ }^{6}$ Author for correspondence: abdiel@cnt.uo.edu.cu
} 
related to the longevity of the species, which is a determining factor in the process of substrate colonization by epiphytes; in some cases, this phenomenon is more evident in the older parts of the thallus (Aguilar-Rosas \& Galindo 1990). In Zostera noltii, Lebreton et al. (2009) found that the number of diatoms was higher on young leaves than on old leaves.

The suitability of macroalgae as substrates for epiphytes can also vary depending on the morphological characteristics of the base; the degree of branching; the roughness and texture of the surface; the production of allelopathic substances such as mucilage; and the thallus growth rate (Lobban \& Harrison 1997). When the thallus surface has little mucilage, it provides favorable conditions for the establishment of epiphytes (Paula \& Oliveira-Filho 1980; Széchy et al. 2006).

In tropical and subtropical regions, studies have shown reductions in the size of communities that develop on rocky shores, especially communities of macroalgae and epiphytes. Such studies have been limited to identifying epiphytic macroalgae species without studying the basic aspects of their ecology (Aguilar et al. 1998; Saad-Navarro \& Riosmena-Rodrígez 2005). Understanding the spatial scales at which the rocky intertidal zone communities vary provides the necessary information to initiate the study of the relative importance of the various factors that can affect these communities and how those factors interact (Underwood et. al. 2000). Therefore, the objective of the present study was to determine the distribution of epiphytic macroalgae on the thallus of their hosts.

\section{Material and methods}

\section{Study area}

The study was conducted in the rocky intertidal zone between the municipalities of Aguadores and Baconao, Cuba, within the Baconao Biosphere Reserve (Managed Resource Protected Area), at eight locations (Fig. 1.): Aguadores 1, Aguadores 2; Sardinero; Juticí; Cajobabo; Verraco, Acuario and Baconao. Located east of the city of Santiago de Cuba, the study zone occupies an area of 5635 hectares of sea on the southeastern coast of the country, between Maisí and Cabo Cruz. It is characterized by a rocky intertidal zone with extensive fields of limestone (surface karst formation).

\section{Sampling design}

Samples were collected on a quarterly basis between June 2010 and March 2011, although inclement weather precluded sample collection in the month of September. Therefore, with the aim of collecting the greatest number of epiphytic species (Lecha \& Chugaev 1989), we performed three samplings: one during the rainy season (in June); and two during the dry season (in December and in March). Samples were collected at low tide in the intertidal zone, which is characterized by short intervals of immersion and emersion.

We collected all epiphytic macroalgae and their hosts in 15 square metal containers $(25 \mathrm{~cm} \times 25 \mathrm{~cm} \times 25 \mathrm{~cm})$ distributed at random (Jover et al. 2012). All macroalgae

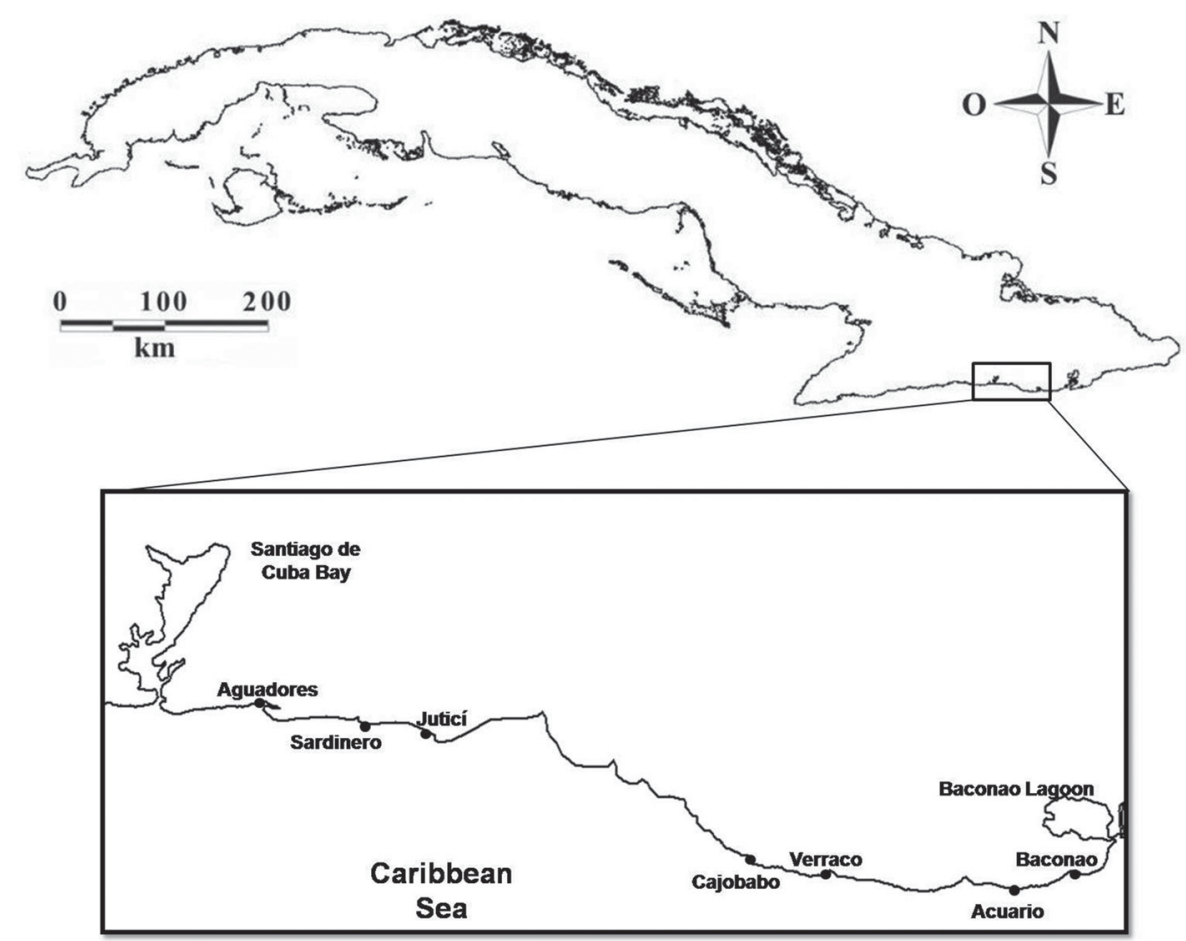

Figure 1. Location of the Aguadores-Baconao coastal sector and of the individual sampling sites. 
that were growing on other macroalgae were classified as epiphytes, according to the criteria of Borowitzka et al. (2006). In accordance with Montañés et al. (2003), the epiphytic macroalgae were separated into two groups by their location on the thallus of the host: at the base or on the surface. The morphofunctional classification of the groups followed the criteria established by Littler \& Littler (1984) and Steneck \& Dethier (1994).

The macroalgae were identified down to the lowest possible taxonomic level on the basis of the specialized literature (Børgesen 1915-20; Taylor 1960; Littler et al. 1989; Littler \& Littler 1997, 2000; Castro et al. 2008; Dawes \& Mathieson 2008; Littler et al. 2008). The species list was created according to the taxonomic criteria established by Wynne (2011). For the higher classes (class, division, and kingdom), we followed the criteria established by Guiry \& Guiry (2011) in Algaebase (http://www.algaebase.org/).

\section{Data analysis}

For each host macroalga and each zone of the thallus, we determined epiphyte species richness on the basis of the number of species observed (Ludwing \& Reynolds 1988).

The origin and significance of differences between the zones of the host thalli were determined by analysis of similarity, with 999,999 permutations applied to the similarity matrix (Clarke \& Warwick 2001). The similarity matrix was derived from the species presence and absence data using the Jaccard index. The principal species responsible for the dissimilarity were identified by similarity percentage analysis (Clarke 1993). These nonparametric multivariate tests were conducted with the statistical program Primer, version 6 (Clarke \& Gorley 2006).

\section{Results}

\section{Epiphytic macroalgae and their hosts: flora}

The observed number in the rocky intertidal zone of the Aguadores-Baconao sector, we observed 102 taxa of epiphytic macroalgae (18 Phaeophyceae, 53 Rhodophyceae and 31 Chlorophyceae), belonging to 14 orders, 25 families and 52 genera. The most well-represented order was Ceramiales, with 30 species, accounting for $29.4 \%$ of the total. Among the Phaeophyceae and Chlorophyceae, respectively, the most well-represented orders were Dictyotales and Cladophorales, with 13 species each.

Among the host macroalgae, we identified 61 taxa: 17 Phaeophyceae, 25 Rhodophyceae and 19 Chlorophyceae, belonging to 14 orders, 23 families and 39 genera. The most well-represented orders were Dictyotales and Ceramiales, with 11 species each. The orders Cladophorales and Siphonocladales presented six species each. The Phaeophyceae genus Dictyota presented the greatest number of species, with six.

\section{Systematic list of epiphytic macroalgae (species listed)}

Domain: Eukaryota

Kingdom: Chromista

Subkingdom: Chromobiota

Division: Heterokontophyta

Class: Phaeophyceae

Order: Dictyotales

Family: Dictyotaceae

Genus: Dictyopteris

1- Dictyopteris delicatula J.V.Lamour.

Genus: Dictyota

2- Dictyota bartayresiana J.V.Lamour.

3- Dictyota ciliolata Sonder ex Kützing

4- Dictyota crenulata J.Agardh

5- Dictyota guineensis (Kützing) P.L.Crouan \& H.M.Crouan

6- Dictyota menstrualis (Hoyt) Schnetter, Hörning \& Weber-Peukert

7- Dictyota mertensii (Martius) Kützing

8- Dictyota pulchella Hörnig \& Schnetter

Genus: Canistrocrpus

9- Canistrocarpus cervicornis (J.V.Lamour.) DePaula \& De Clerck

10- Canistrocarpus crispatus (J.V.Lamour.) De Paula \& De Clerck

Genus: Lobophora

11- Lobophora variegata (J.V.Lamour.) Womersley ex E.C.Oliveira

Genus: Padina

12- Padina sanctae-crucis Børgesen 
13- Padina gymnospora (Kützing) Sonder

Order: Sphacelariales Family: Sphacelariaceae

Genus: Sphacelaria

14- Sphacelaria novae-hollandiae Sonder

Order: Ectocarpales Family: Scytosiphonaceae

Genus: Chnoospora

15- Chnoospora minima (Hering) Papenfuss

Order: Fucales

Family: Sargassaceae

Genus: Sargassum

16- Saregassum buxifolium (J.Agardh) M.J.Wynne

17- Sargassum polyceratium Montagne

Genus: Turbinaria

18- Turbinaria tricostata E.S.Barton

Kingdom: Plantae

Subkingdom: Biliphyta

Division: Rhodophyta

Class: Florideophyceae

Order: Corallinales

Family: Hapalidiaceae

19- Mesophyllum sp.

Genus: Mesophyllum

Family: Corallinaceae

Genus: Hydrolithon

20- Hydrolithon farinosum (J.V.Lamour.) D.Penrose \& Y.M.Chamberlain

Genus: Jania

21- Jania adhaerens J.V.Lamour.

22- Jania capillacea Harvey

23- Jania cubensis Montagne ex Kützing

24- Jania rubens (Linnaeus) J.V.Lamour.

Genus: Amphiroa

25- Amphiroa beauvoisii J.V.Lamour.

26- Amphiroa fragilissima (Linnaeus) J.V.Lamour.

27- Amphiroa rigida J.V.Lamour.

Order: Ceramiales

Family: Ceramiaceae

Genus: Ceramium

28- Ceramium brasiliense A.B.Joly

29- Ceramium brevizonatum var. caraibicum H.E.Petersen \& Børgesen

30- Ceramium cimbricum H.E.Petersen

31- Ceramium codii (H.Richards) Mazoyer

32- Ceramium comptum Børgesen

33- Ceramium corniculatum Montagne

34- Ceramium dawsonii A.B.Joly

35- Ceramium sp.

Genus: Gayliella

36- Gayliella transversalis (Collins \& Hervey) T.O.Cho \& Fredericq

Genus: Centroceras

37- Centroceras clavulatum (C.Agardh) Montagne

Family: Wrangeliaceae

Genus: Griffithsia

38- Griffithsia globulifera Harvey ex Kützing

Genus: Ptilothamnion 
39- Ptilothamnion speluncarum (Collins \& Hervey) D.L.Ballantine \& M.J.Wynne

Family: Dasyadaceae

Genus: Dasya

40- Dasya sp.

Genus: Heterosiphonia

41- Heterosiphonia crispella (C.Agardh) M.J.Wynne

Family: Rhodomelaceae

Genus: Chondrophycus

42- Chondrophycus iridescens (M.J. Wynne \& D.L. Ballantine) Garbary \& Harper

Genus: Palisada

43- Palisada perforata (Bory) K.W.Nam

44- Laurencia caraibica P.C.Silva

Genus: Laurencia

45- Laurencia obtusa (Hudson) J.V.Lamour.

46- Laurencia sp.

Genus: Digenia

47- Digenea simplex (Wulfen) C.Agardh

Genus: Lophosiphonia

48- Lophosiphonia cristata Falkenberg

Genus: Herposiphonia

49- Herposiphonia bipinnata M.Howe

50- Herposiphonia secunda (C.Agardh) Ambronn

Genus: Neosiphonia

51- Neosiphonia sphaerocarpa (Børgesen) M.S.Kim \& I.K.Lee

52- Neosiphonia howei (Hollenberg) Skelton \& G.R. South

Genus: Polysiphonia

53- Polysiphonia atlantica Kapraun \& J.N.Norris

54- Polysiphonia havanensis Montagne

55- Polysiphonia schneideri Stuercke \& Freshwater

56- Polysiphonia scopulorum Harvey

57- Polysiphonia scopulorum var. villum (J.Agardh) Hollenberg

Order: Gelidiales

Family: Gelidiaceae

Genus: Gelidium

58- Gelidium americanum (W.R.Taylor) Santelices

59- Gelidium pusillum (Stackhouse) Le Jolis

Family: Gelidiellaceae

Genus: Gelidiella

60- Gelidiella acerosa (Forsskål) Feldmann \& G.Hamel

Genus: Parviphycus

61- Parviphycus setaceus (Feldmann) Afonso-Carrillo, Sansón, Sangril \& Díaz-Villa

62- Parviphycus trinitatensis (W.Rtaylor) M.J.Wynne

Order: Gigartinales

Family: Cystocloniaceae

Genus: Hypnea

63- Hypnea musciformis (Wulfen) J.V.Lamour.

64- Hypnea spinella (C.Agardh) Kützing

65- Hypnea valentiae (Turner) Montagne

Order: Rhodymeniales

Family: Rhodymeniales

Genus: Botryocladia

66- Botryocladia pyriformis (Børgesen) Kylin

Family: Champiaceae

Genus: Champia

67- Champia parvula (C.Agardh) Harvey 
8- Champia vieillardii Kützing

Family: Lomentariaceae

Genus: Ceratodictyon

69- Ceratodictyon intricatum (C.Agardh) R.E.Norris

70- Ceratodictyon scoparium (Montagne \& Millardet) R.E.Norris

71- Ceratdictyon variable (Grefville ex J.Agardh) R.E.Norris

Kingdom: Plantae

Subkingdom: Viridaeplantae

Division: Chlorophyta

Class: Ulvophyceae

Order: Ulvales

Family: Ulvaceae

Genus: Pringsheimiella

72- Pringsheimiella scutata (Reinke) Marchewianka

Genus: Ulva

73- Ulva fasciata S.F.Gray

74- Ulva flexuosa Wulfen

75- Ulva flexuosa subsp. paradoxa (C.Agardh) M.J.Wynne

76- Ulva lactuca Linnaeus

77- Ulva rigida C.Agardh

Order: Cladophorales

Family: Anadyomenaceae

Genus: Anadyomene

78- Anadyomene stellata (Wulfen) C.Agardh

Family: Cladophoraceae

Genus: Cladophora

79- Cladophora albida (Nees) Kutzing

80- Cladophora brasiliana G.Martens

81- Cladophora cf. liniformis Kützing

82- Cladophora prolifera (Roth) Kützing

83- Cladophora vagabunda (Linnaeus) C.Hoek

84- Cladophora sp.

Genus: Chaetomorpha

85- Chaetomorpha aerea (Dillwyn) Kützing

86- Chaetomorpha antennina (Bory) Kützing

87- Chaetomorpha clavata Kützing

88- Chaetomorpha gracilis Kützing

89- Chaetomorpha vieillardii (Kützing) M.J.Wynne

Genus: Bryobesia

90- Bryobesia johannae Weber-van Bosse

Order: Siphonocladales

Family: Boodleaceae

Genus: Cladophoropsis

91- Cladophoropsis macromeres W.R.Taylor

92- Cladophoropsis membranacea (Bang ex C.Agardh) Børgesen

Genus: Boodlea

93- Boodlea struveoides M.A. Howe

Genus: Phyllodictyon

94- Phyllodictyon anastomosans (Harvey) Kraft \& M.J.Wynne

Family: Siphonocladaceae

Genus: Dictyosphaeria

95- Dictyosphaeria ocellata (M.Howe) J.L.Olsen-Stojkovich

Family: Valoniaceae

Genus: Valoniopsis

96- Valoniopsis pachynema (G.Martens) Børgesen

Genus: Valonia 
97- Valonia ventricosa J.Agardh

Class: Bryopsidophyceae

Order: Bryopsidales

Family: Bryopsidaceae

Genus: Bryopsis

98- Bryopsis plumosa (Hudson) C.Agardh

Family: Udoeaceae

Genus: Boodleopsis

99- Boodleopsis pusilla (F.S.Collins) W.R.Taylor, A.B.Joly \& Bernatowicz

Family: Halimedaceae

Genus: Halimeda

100- Halimeda sp.

Class: Dasycladophyceae

Order: Dasycladales

Family: Polyphysaceae

Genus: Acetabularia

101- Acetabularia crenulata J.V.Lamour.

Genus: Parvocaulis

102- Parvocaulis polyphysoides (P.Crouan \& H.Crouan) S. Berger, U. Fettweiss, S. Gleissberg, L. B. Liddle, U. Richter, H. Sawitsky, H. \& G. C. Zuccarello

\section{Distribution of epiphytic macroalgae on the host thalli}

The distribution of epiphytes on the host thalli varied considerably. In 31 host species, the number epiphyte species was higher on the surface of the thallus than at its base, whereas the inverse was observed in 25 , and the number epiphyte species was comparable between the two zones in five host species. Epiphytes were located only on the surface of the thallus in nine host species and only at its base in three. The cumulative species richness (both zones) was 89 species. The greatest epiphyte species richness was recorded for macroalgae with thalli that were more complex, belonging to the corticated, leathery, leafy and globose morphofunctional groups (Tab. 1).

The total $\mathrm{R}$ value for the comparison between the two areas was $-0.001(\mathrm{p}=0.398)$. The mean dissimilarity between the surface and the base of the host thallus, in terms of the species composition of epiphytic macroalgae, was $96.64 \%$. The species Hydrolithon farinosum (J.V.Lamouroux) D.Penrose \& Y.M.Chamberlain and Polysiphonia atlantica Kapraun \& J.N.Norris accounted for $43.76 \%$ of the dissimilarity.

The species that accounted for the majority of the mean similarity among epiphytic macroalgae at the base of the host thallus $(2.99 \%)$ was Hydrolithon farinosum, which accounted for $59.36 \%$. In addition, $H$. farinosum accounted for $92.37 \%$ of the mean similarity among epiphytic macroalgae on the surface of the host thallus (4.51\%).

Among the species occurring at the base of the thallus, the following were the most common: Dictyosphaeria ocellata (M.Howe) J.L.Olsen-Stojkovich ( $\mathrm{n}=11)$; Cladophora albida (Nees) Kutzing ( $\mathrm{n}=13)$; Valoniopsis pachynema (G.Martens) Børgesen ( $\mathrm{n}=19)$; Hypnea spinella (C.Agardh) Kützing ( $\mathrm{n}=19)$; Palisada perforata (Bory) K.W.Nam
( $\mathrm{n}=10)$; Centroceras clavulatum (C.Agardh) Montagne ( $\mathrm{n}=11)$; Amphiroa rigida J.V.Lamouroux ( $\mathrm{n}=18)$; Amphiroa fragilissima (Linnaeus) J.V.Lamouroux ( $\mathrm{n}=12)$; Jania adhaerens J.V.Lamouroux $(\mathrm{n}=21)$; Hydrolithon farinosum (n = 39); and Dictyota menstrualis (Hoyt) Schnetter, Hörning $\&$ Weber-Peukert $(n=10)$. There were 20 host species that showed no epiphytes at the base of the thallus.

The following were the most common species occurring on the surface of the thallus: Padina sanctae-crucis Børgesen $(\mathrm{n}=12)$; Hydrolithon farinosum $(\mathrm{n}=40)$; Centroceras clavulatum $(\mathrm{n}=15)$; Ceramium brasiliense A.B.Joly $(\mathrm{n}=11)$; Neosiphonia sphaerocarpa (Børgesen) M.S.Kim \& I.K.Lee (n $=10)$; Polysiphonia atlantica $(\mathrm{n}=18)$; and Cladophora albida $(\mathrm{n}=10)$. There were only three host species that showed no epiphytes on the surface of the thallus (Tab. 1): Gelidium americanum (W.R.Taylor) Santelices; Ulva flexuosa Wulfen; and Bryopsis plumosa (Hudson) C.Agardh.

\section{Discussion}

\section{Epiphytic macroalgae and their hosts: typical flora}

The 102 taxa of epiphytic macroalgae observed here correspond to $21 \%$ of those identified for the waters of Cuba, whereas the host species correspond to 12.5\% (Suárez 2005). The level of species richness observed here was high in comparison with those previously reported for Cuban waters. That might be due, in part, to the community approach taken in our study of the hosts, given that the majority of investigations of the hosts of epiphytic macroalgae have been conducted at the population level.

In an estuarine ecosystem, Cabrera et al. (2005) identified only 25 epiphytes on Palisada perforata. In contrast, 
Table 1. Characteristics of the macroalgae hosting epiphytic macroalgae, including morphofunctional group, thallus zone in which the epiphytes were found, and epiphyte species composition, in a rocky intertidal zone in the Baconao Biosphere Reserve, in Cuba.

\begin{tabular}{|c|c|c|c|}
\hline $\begin{array}{l}\text { Division } \\
\text { Species }\end{array}$ & MG & Thallus zone & Epiphyte species* \\
\hline \multicolumn{4}{|l|}{ HETEROKONTOPHYTA } \\
\hline \multirow{2}{*}{ Dictyopteris delicatula } & \multirow{2}{*}{ LG } & B & $19,20,59,63,64,65,70,76,86,96,98$ \\
\hline & & S & 20 \\
\hline \multirow{2}{*}{ Dictyota bartayresiana } & \multirow{2}{*}{ LG } & B & 20 \\
\hline & & S & $20,28,34,57$ \\
\hline \multirow{2}{*}{ Dictyota cervicornis } & \multirow{2}{*}{ LG } & B & $20,21,26,37,63,64$ \\
\hline & & S & 20 \\
\hline \multirow{2}{*}{ Dictyota ciliolata } & \multirow{2}{*}{ LG } & B & 12,20 \\
\hline & & S & 20,53 \\
\hline \multirow{2}{*}{ Dictyota guineensis } & \multirow{2}{*}{ LG } & B & 20,53 \\
\hline & & S & 20 \\
\hline \multirow{2}{*}{ Dictyota menstrualis } & \multirow{2}{*}{ LG } & B & $12,20,21,22,31,32,33,43,45,51,64,96,80$ \\
\hline & & S & 20 \\
\hline \multirow{2}{*}{ Dictyota mertensii } & \multirow{2}{*}{ LG } & B & $6,11,20,21,26,43,50,52,78,79,84$ \\
\hline & & S & 20,53 \\
\hline \multirow{2}{*}{ Dictyota pulchella } & \multirow{2}{*}{ LG } & B & 20 \\
\hline & & S & 20,53 \\
\hline Lobophora variegata & Lthy & S & 69 \\
\hline \multirow{2}{*}{ Padina gymnospora } & \multirow{2}{*}{ Lthy } & B & $21,27,37,64,73,74,76,80,91,97$ \\
\hline & & S & 74 \\
\hline \multirow[t]{2}{*}{ Padina sanctae-crucis } & \multirow[t]{2}{*}{ Lthy } & B & $\begin{array}{l}5,6,7,8,20,21,22,26,27,28,30,31,32,34,37,38,39,40,41,43,44,45,46, \\
47,50,51,53,55,56,63,64,78,79,81,82,84,87,89,91,92,93,95,96,97,100\end{array}$ \\
\hline & & S & $20,28,36,37,38,39,40,41,49,50,51,55,56,57,64,67,79,88$ \\
\hline \multirow{2}{*}{ Chnoospora minima } & \multirow{2}{*}{ Cort } & B & 20,96 \\
\hline & & S & 20 \\
\hline Sargassum buxifolium & Lthy & B & $\begin{array}{l}2,5,6,7,9,11,12,18,20,21,23,25,26,27,32,42,43,44,45,51,52,53,63,67,68 \text {, } \\
69,71,78,80,84,88,95,96\end{array}$ \\
\hline & & S & $7,20,51,53,89,91$ \\
\hline Sarrassum fluitans & Lthy & B & $20,25,27,50,60,64,95$ \\
\hline & Lthy & S & 20,95 \\
\hline Sarrossum polvceratium & Ithy & B & $2,11,12,17,25,27,28,29,30,37,43,44,45,51,60,63,64,70,76,79,88,95,96$ \\
\hline sargassum polyceratium & Leny & S & $1,7,17,19,20,35,36,45,52,51,53,98$ \\
\hline Turbinaria tricostata & Lthy & B & $\begin{array}{l}2,6,7,11,12,20,21,23,25,26,27,37,29,31,35,38,39,40,43,44,45,53,63,64,66 \text {, } \\
69,71,78,79,84,95,96\end{array}$ \\
\hline & & S & $20,27,41,49,50,52,54,96$ \\
\hline Tuebinaria turbinato & Lthy & B & $2,6,20,27,45,53,64,79,90,96$ \\
\hline Turbinaria turbinata & & S & 20 \\
\hline RHODOPHYTA & & & \\
\hline Jania cubensis & $\mathrm{AC}$ & S & 32 \\
\hline Amphiroa rigida & $\mathrm{AC}$ & S & 78 \\
\hline Tricleocarpa cilindrica & $\mathrm{AC}$ & S & $32,53,68,90$ \\
\hline Centroceres sn & Fila & B & 20 \\
\hline Centroceras sp. & & S & 20,50 \\
\hline Acanthothora muscoides & Cort & B & $21,27,64,96$ \\
\hline Acanthophora muscoldes & & S & 98 \\
\hline Chondrothycus iridiscens & Cort & B & 20,95 \\
\hline Chondrophycus tridiscens & & $S$ & $6,7,20,21,53,64,93$ \\
\hline Yuzurua poiteaui & Cort & B & $20,51,79$ \\
\hline ruzurua porteaul & & S & $20,32,53$ \\
\hline
\end{tabular}


Table 1. Continuation

\begin{tabular}{|c|c|c|c|}
\hline \multirow{2}{*}{$\begin{array}{l}\text { Division } \\
\text { Species }\end{array}$} & MG & Thallus zone & \multirow[t]{2}{*}{ Epiphyte species* } \\
\hline & & & \\
\hline \multirow{2}{*}{ Palisada perforata } & Cort & B & $\begin{array}{l}2,6,7,11,12,19,20,21,25,26,27,28,31,32,38,41,44,45,46,49,51,60,61,62,63 \text {, } \\
67,78,84,89,95,96,97,101\end{array}$ \\
\hline & & $S$ & $\begin{array}{l}2,3,6,8,12,15,17,20,23,25,26,27,28,30,31,34,35,37,44,49,50,51,52,53,54, \\
60,63,64,69,70,78,79,91,92,95,96,99,101\end{array}$ \\
\hline \multirow[b]{2}{*}{ Digenea simplex } & Cort & B & $20,21,25,26,43,44,64,79,95,99$ \\
\hline & & S & $\begin{array}{l}6,7,10,12,14,16,19,20,22,28,37,43,45,46,48,49,51,53,54,56,47,62,79,82, \\
83,85,88,91,93,96\end{array}$ \\
\hline Herposiphonia bipinnata & Fila & S & 11 \\
\hline \multirow{2}{*}{ Laurencia caraibica } & Cort & B & $6,20,51,79$ \\
\hline & & S & $6,20,21,29,50,53,70$ \\
\hline \multirow{2}{*}{ Laurencia obtusa } & Cort & B & $2,6,12,20,27,53,55,57,69,70,91$ \\
\hline & & $S$ & $4,11,20,28,32,37,50,51,53,81$ \\
\hline \multirow{2}{*}{ Laurencia sp. } & Cort & B & $12,20,21,96$ \\
\hline & & S & $8,12,15,20,23,53,79,80,91$ \\
\hline \multirow{2}{*}{ Neosiphonia sphaerocarpa } & Fila & B & $6,20,33,79$ \\
\hline & & S & $12,20,79$ \\
\hline \multirow{2}{*}{ Polysiphonia scopolorum } & Fila & B & 20 \\
\hline & & S & $20,37,79,84$ \\
\hline Gelidium americanum & Fila & B & 21 \\
\hline \multirow{2}{*}{ Gelidium pusillum } & Fila & B & $21,27,58,64,86,91,95,96,98$ \\
\hline & & S & 19,28 \\
\hline \multirow{2}{*}{ Gelidiella acerosa } & Cort & B & 20,25 \\
\hline & & S & $20,37,92$ \\
\hline \multirow{2}{*}{ Hypnea musciformis } & Cort & B & $20,21,26,27,53,96$ \\
\hline & & S & $1,20,28,37,51,54,56,57,74,75,76,83,96,98$ \\
\hline \multirow{2}{*}{ Hypnea spinella } & Cort & B & $2,12,20,21,26,30,37,43,79,86,95,96$ \\
\hline & & S & $1,6,11,12,13,20,37,43,45,51,56,59,74,76,77,82,83,88,89,91,98$ \\
\hline \multirow{2}{*}{ Hypnea valentiae } & Cort & B & $21,74,96$ \\
\hline & & S & $1,37,76,98$ \\
\hline Ochtodes secundiramea & Fila & S & 98 \\
\hline \multirow{2}{*}{ Champia parvula } & Fila & B & 20 \\
\hline & & S & 20 \\
\hline \multirow{2}{*}{ Ceratodyction intricatum } & Cort & B & 20 \\
\hline & Cort & S & 12,20 \\
\hline \multirow{2}{*}{ Ceratodyction scoparia } & Cort & B & $1,21,27,37,63,64,74,76,77,79,86,91,98$ \\
\hline & Cort & S & 1 \\
\hline \multicolumn{4}{|l|}{ CHLOROPHYTA } \\
\hline Ulva flexuosa & LG & B & 37 \\
\hline \multirow{2}{*}{ Ulva lactuca } & LG & B & $1,13,19,21,22,26,27,37,59,65,74,80,86,89,91,94,96,98$ \\
\hline & & S & 20 \\
\hline \multirow{2}{*}{ Anadyomene stellata } & LG & B & $11,20,27,43,44,45,56$ \\
\hline & LU & S & $11,20,53$ \\
\hline \multirow[b]{2}{*}{ Valoniopsis pachynema } & & B & $20,24,25,26,27,32,43,44,46,53,56,62,64,78,83,95$ \\
\hline & Fila & S & $\begin{array}{l}2,5,6,8,9,11,12,14,20,26,27,35,43,45,47,53,55,57,58,63,64,73,73,75,76,77 \text {, } \\
77,78,79,80,88,89,91,95,98\end{array}$ \\
\hline \multirow{2}{*}{ Chaetomorpha antennina } & Fila & B & $21,32,37,51,59,76,78,80$ \\
\hline & $171 d$ & S & $37,64,74,76$ \\
\hline \multirow{2}{*}{ Chaetomorpha vieillardii } & Fila & B & $2,59,64,73,76$ \\
\hline & & S & 98 \\
\hline
\end{tabular}


Table 1. Continuation.

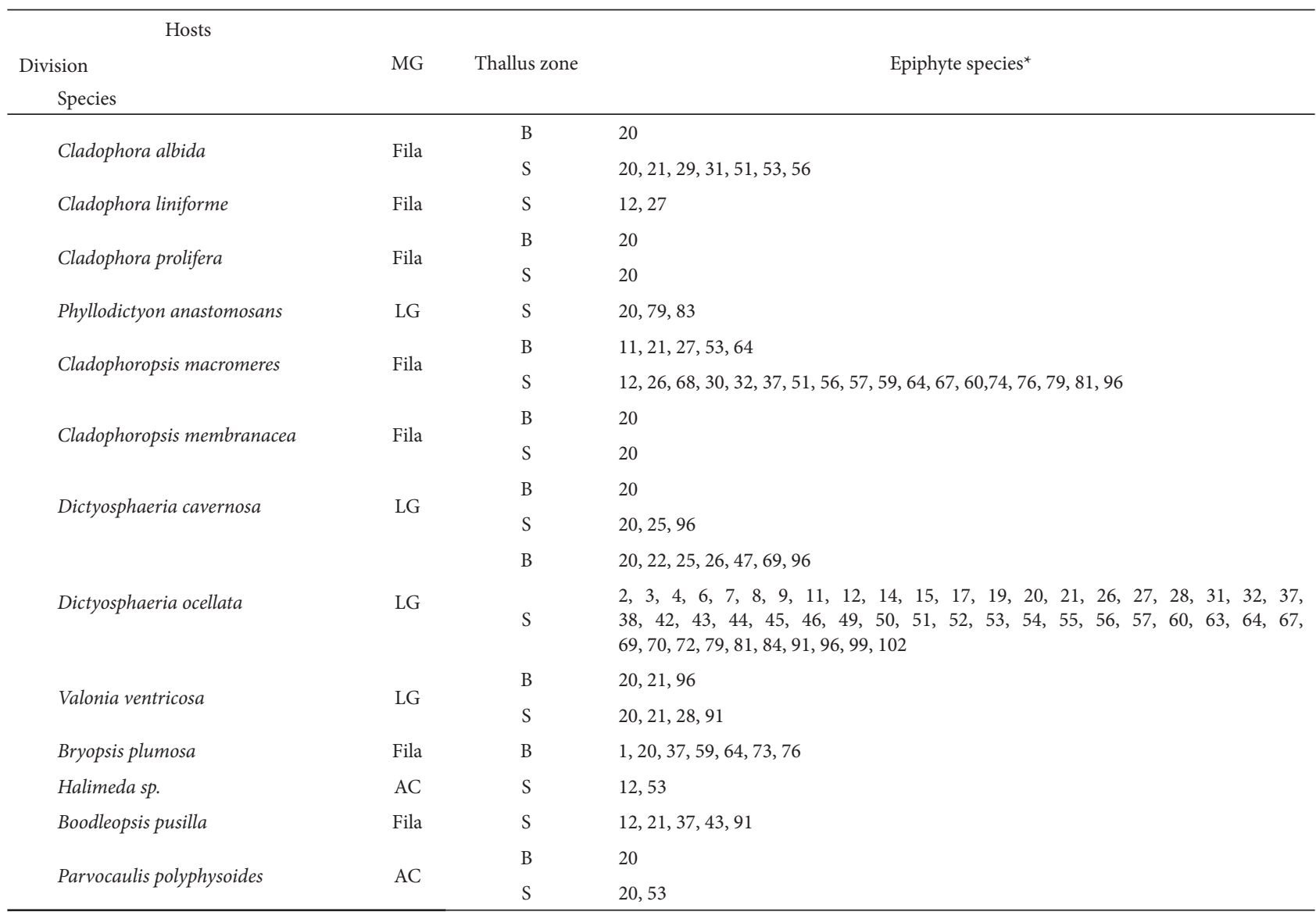

MG - morphofunctional group; LG - leafy, globose; Lthy - leathery; Cort - corticate; AC - articulated calcareous; Fila -filamentous.

${ }^{*}$ For the names of the species corresponding to the numbers shown, see the taxonomic list in the Results section.

Suárez et al. (1989) identified 98 species of epiphytic macroalgae on Stypopodium zonale (Lamouroux) Papenfuss in a rocky subtidal ecosystem. That discrepancy can be explained by the differences between the two host species, in terms of the complexity of the thallus (greater in S. zonale) and in terms of habitat (species richness is typically greater in rocky subtidal ecosystems than in estuarine ecosystems).

Zayas et al. (2006) found only 13 species of epiphytic macroalgae within the community of macroalgae in the reef lagoon at Guardalavaca Beach. That low level of species richness could be attributed to the fact that epiphytic macroalgae more adhere to Thalassia testudinum Banks \& Sol. ex K.D.Koenig, in algal beds, or to angiosperms (Borowitzka et al. 2006).

The greater epiphyte richness observed among hosts belonging to the morphofunctional groups with higher thallus complexity is due to the fact that those groups present morphological differences among their component species, which makes them responsible for increasing the spatial heterogeneity of the community. In addition, species with differentiated blades and thalli have greater morphological complexity, are more resistant to adverse conditions and confer greater stability upon the community (Littler \& Littler 1980; 1984).

\section{Distribution of epiphytic macroalgae on the host thallus}

Greater epiphyte species richness on the surface of the thallus might be determined by the protection that provides against the extreme conditions in the intertidal zone, such as desiccation and wave exposure, as well as avoiding the competition for light that could lead to the overlapping of host thalli. In turn, macroalgae hosts with more epiphytes at the thallus base could better withstand those adversities and tolerate competition for light. The various structures that give rise to the thalli differ in terms of desiccation and solar irradiation. Longtin et al. (2009) found that these two factors were more intense in the distal segments of the thallus than at its base.

The variation in the accumulated epiphyte species richness in the rocky intertidal zone at the Aguadores-Baconao site is related to the complexity, rather than the zonation, of the host thalli. Montañés et al. (2003) argued that not only the habits but also the organizational type of epiphytes directly conditions their adhesion sites and preference for 
different parts of the host. The morphology of the host thallus is also crucial, it is expected that structurally complex thalli favor colonization by a greater number of epiphytes, because they create a greater number of niches and shelters (Ayala \& Martín 2003). Apparently morphologically complex thalli, such as those of leathery corticate algae, create protected microhabitats (Kendrick \& Burt 1997; Lavery \& Vanderklift 2002). In addition, the texture and structure of the thalli are frequently used by epiphytes, increasing the complexity of the habitat (Hacker \& Steneck 1990).

The fact that the similarity in overall shape was near zero indicates no difference in the distribution of epiphytic macroalgae, which might be attributable to the similarity between the base of the thallus and its surface, in terms of epiphyte species richness. However, the dissimilarity values are very high, given the existence of quite significant differences in epiphyte species composition and in the proportional contributions of individual species.

Species such as Hydrolithon farinosum, Centroceras clavulatum and Cladophora albida showed no preference for any specific area of the host thallus, presenting high values of frequency of appearance at its base and on its surface. This behavior could be determined by the low complexity of the host thalli, given the possibility that the developmental requirements of those species might not be as specific as are those of other epiphytes.

The hosts that did not have epiphytes adhering to the thallus base were typically the simpler forms, with few occurrences during the sampling periods. Macroalgae that host a great number of epiphytes in general or at the thallus base might have greater viability in the ecosystem, as has been suggested by various authors (Davis et al. 1989; Wahl 1989). The significantly low number of species hosting epiphytes of the thallus surface might be attributable to the epiphytes having a preference for zones that provide more light and more nutrients (Pedersen \& Borum 1996; Lobban \& Harrison 1997; Kraberg \& Norton 2007). Ulva flexuosa and Bryopsis plumosa both present thalli that are structurally simple, with very smooth blade surfaces.

We conclude that the development of epiphytes can be influenced not only by the extreme physical conditions in the rocky intertidal zone but also by the structural characteristics of the host thalli and of the epiphytes, their ability to adhere to other macroalgae thalli or allow their own thalli to be adhered to by others.

\section{Acknowledgments}

The authors would like to thank Michael Wynne, for the corrections to the taxonomic nomenclature, and Humberto Morris, for the translation of the abstract to Portuguese and for the unconditional assistance. The authors are also grateful to Gary Kendrick and Lizette Quan for their help in constructing the bibliography, as well as to the peer reviewers, whose comments and suggestions resulted in substantial improvement of the manuscript.

\section{References}

Aguilar, M.; Aguilar-Rosa L. \& Aguilar-Rosas R. 1998. Algas marinas de la región central del Estado de Quintana Roo, México. Polibotánica 7: 15-32.

Aguilar-Rosas, R. \& Galindo A. 1990. Ecological aspects of Sargassum muticum (Fucales, Phaeophyta) in Baja California: reproductive phenology and epiphytes. Hydrobiologia 204/205: 185-190.

Ang, P. \& DeWreede R. 1992. Density-dependence in a population of Fucus distichus. Marine Ecology Progress Series 90: 169-181.

Ayala, Y. \& A. Martin. 2003. Relaciones entre la comunidad de anfípodos y las macroalgas a las que están asociados, en una plataforma rocosa del litoral central de Venezuela. Boletino Del Instituto Espanol De Oceanografia 19: 171.184.

Børgesen, F. 1915-20. II. Rhodophyceæ, with addenda to the Chlorophyceæ, Phæophyceæ and Rhodophyceæ. Dansk Botanisk Arkiv 3: 1-504.

Borowitzka, M.A.; Lavery, P.S. \& van Keulen, M. (2006) Epiphytes of seagrasses, Pp. 441-461. In: Larkum, A.W.D.; Orth, P.J. \& Duarte, C.M. (Eds.). Seagrasses: Biology, Ecology and Conservation. Berlín, Springer.

Brawley, S. \& Johnson L. 1992. Gametogenesis, gametes and zygotes: an ecological perspective on sexual reproduction in the algae. British Phycological Journal 27: 233-252.

Bruno, J.; Stachowicz J. \& Bertness M. 2003. Inclusion of facilitation into ecological theory. Trends in Ecology and Evolution 18(3): 119-125.

Cabrera, R.; Moreira, Á.; Primelles, J. \& Suárez, A. 2005. Variación de la Biomasa de Chondrophycus papillosus (C. Agardh) Garvary et Harper (Ceramiales: Rhodophyta) y su epifitismo en la Bahía de Nuevitas, Cuba. Revista de Investigaciones Marinas 26(1): 15-20.

Castro, J. de; Barros-Barreto, M. \& Pita, S. 2008. A família Ceramiaceae (Ceramiales, Rhodophyta) no estado da Bahia, Brasil. Pp. 75-159. En: A. Santíes y K. Dreckmann (Eds.). Monografías Ficológicas 3. Universidad Autónoma de México.

Clarke, K. 1993. Non-parametric multivariate analyses of changes in community structure. Australian Journal of Ecology 18: 117-143.

Clarke, K. \& Gorley, R. 2006. PRIMER v 6: User manual/tutorial, PRIMER-E. United Kingdom, Plymouth.

Clarke, K. \& Warwick, R. 2001. Change in marine communities: an approach to statistical analysis and interpretation. PRIMER-E. United Kingdom, Plymouth.

Davis, A.; Targett, N.; McConnell, O. \& Young, C. 1989. Epibiosis of marine algae and benthic invertebrates: natural product chemistry and other mechanisms inhibiting settlement and overgrowth. Pp. 85-114. In: Scheuer, P. (Ed.). Bioorganic Marine Chemistry. V. 3. Berlin, Springer-Verlag.

Dawes, C. \& Mathieson, A. 2008. The Saeweeds of Florida. Florida, University of Florida Press.

Foster, M. 1990. Organization of macroalgal assemblages in the Northeast Pacific: the assumption of homogeneity and the illusion of generality. Hydrobiologia 192: 21-34.

Foster, M. 1991. Rammed by the Exxon Valdez: a reply to Paine. Oikos 62: $93-96$

Guiry, M. \& Guiry, G. 2011. AlgaeBase. World-wide electronic publication, National University of Ireland, Galway. http://www.algaebase.org. (Consultado: 16 mayo 2011).

Hacker, S. \& Steneck R. 1990. Habitat architecture and body- size-dependent habitat selection of a phytal amphipod. Ecology 71: 2269-2285.

Jover, A.; Reyes, L.; Gómez, L. \& Suárez, A. 2012. Variación espacial y temporal de las macroalgas del mesolitoral rocoso en Aguadores-Baconao, Cuba I: Composición. Revista de Investigaciones Marinas 32: 38-49.

Kraberg, A. \& Norton, T. 2007. Effect of epiphytism on reproductive and vegetative lateral formation in the brown, intertidal seaweed Ascophyllum nodosum (Phaeophyceae). Phycological Research 55: 17-24.

Kendrick G. \& Burt, J. 1997. Seasonal changes in epiphytic macro-algae assemblages between offshore exposed and inshore protected Posidonia sinuosa Cambridge et Kuo seagrass meadows, Western Australia. Botanica Marina 40: 77-85

Lavery P. \& Vanderklift, M. 2002. A comparison of spatial and temporal patterns in epiphytic algae assemblages of the seagrasses Amphibolis griffithii and Posidonia coriacea. Marine Ecology Progress Series 236: 99-112 
Lebreton, B.; Richard P.; Radenac G.; Bordes M.; Bréret M.; Arnaud C.; Mornet F. \& Blanchard G. 2009. Are epiphytes a significant component of intertidal Zostera noltii beds? Aquatic Botany 91: 82-90.

Lecha, L. \& Chugaev, A. 1989. La bioclimatología y algunas de sus aplicaciones en condiciones de clima tropical húmedo. La Habana, Editorial Academia, Cuba.

Little, C. \& Kitching J. 1996. The biology of rocky shores. Oxford, Oxford University Press.

Littler, M.M. \& D.S. Littler. 1980. The evolutions of thallus form and survival strategies in benthic marine macroalgae: field and laboratory test of and functional: forms model. American Naturalist 116(1): 25-44.

Littler, D.S. \& M.S. Littler. 1984. Relationships between macoalgal functional form groups and substrata stability in a subtropical rocky-intertidal system. Journal of Experimental Marine Biology and Ecology 74: 13-34.

Littler, D. \& Littler, M. 1997. An illustrated marine flora of the Pelican Cays, Belize. Boletim de Botânica 9: 1-149.

Littler, D. \& Littler, M. 2000. Caribbean Reef Plants. An identification to tha reef plants of the Caribbean, Bahamas, Florida and Gulf of Mexico. Florida, Offshore Graphics, Inc.

Littler, D.; Littler, M. \& Dennis, M. 2008. Submersed Plants of the Indian River Logoon. Florida, Offshore Graphics, Inc.

Littler, D.; Littler, M.; Rucher, K. \& Noris, J. 1989. Marine Plants of the Caribbean. A fiel guide from Florida to Brazil. New York, Smithosonian Institution Press.

Lobban, C. \& Harrison P. 1997. Seaweed ecology and physiology. Cambridge, Cambridge University Press.

Longtin, C. \& Scrosati, R. 2009. Role of surface wounds and brown algal epiphytes in the colonization of Ascophyllum nodosum (Phaeophyceae) fronds by Vertebrata lanosa (Rhodophyta). Journal of Phycolgy 45: 535-539.

Longtin, C.; Scrosati, R.; Whalen, G. \& Garbary, D. 2009. Distribution of algal epiphytes across environmental gradients at different scales: intertidal elevation, host canopies and host fronds. Journal of Phycolgy 45: 820-827.

Ludwig, J. \& Reynolds, J. 1988. Statistical Ecology. Nueva York, John Wiley and Sons.

Martin-Smith, K. 1993. Abundante of mobile epifauna: the role of habitat complexity and predation by fishes. Journal of Experimental Marine Biology and Ecology 174: 243-260.

Montañes, M.; Reyes, J. \& Senzón, M. 2003. La comunidad de epífitos de Zonaria tournefortiien el norte de Tenerife (islas Canarias): análisis florístico y comentarios sobre su epifauna. Vieraea 31: 121-132.

Paine, R. 1991. Between Scylla and Charybdis: do some kinds of criticism merit a response? Oikos 62: 90-92.

Paula, E. \& Oliveira-Filho E. 1980. Phenology of two populations of Sargassum cymosum (Phaeophyta- Fucales) of São Paulo State coast, Brazil. Boletim de Botânica 8: 21-39.
Pedersen, M. \& Borum, J. 1996. Nutrient control of algal growth in estuarinen waters. Nutrient limitation and the importance of nitrogen requirements and nitrogen storage among phytoplankton and species of macroalgae. Marine Ecology Progress Series 142: 261-272.

Reed, D. 1990a. The effects of variable settlement and early competition on patterns of kelp recruitment. Ecology 71(2): 776-787.

Reed, D. 1990b. An experimental evaluation of density dependence in a subtidal algal population. Ecology 71: 2286-2296.

Saad-Navarro, G. \& Riosmena-Rodrígez R. 2005. Variación espacial y temporal de la riqueza florística de macroalgas en la zona rocosa de Bahía de Muertos, B.C.S. México. Ciencia y Mar IX (26): 19-32.

Steneck, R.S. \& M.N. Dethier. 1994. A functional group approach to the structure of algal dominated communities. Oikos 69: 476-498.

Suárez, A. 2005. Lista de las macroalgas marinas cubanas. Revista de Investigaciones Mararinas 26(2): 93-148.

Suárez, A.; Gil, L. \& Poseck R. 1989. Variación del epifitismo en Stypopodium zonale (Lamouroux) Papenfuss a lo largo de un año. Revista de Investigaciones Mararinas 10(1): 3-20.

Széchy, M.; Galliez M. \& Marconi M. 2006. Quantitative variables applied to phenological studies on Sargassum vulgare C. Agardh (Phaeophyceae, Fucales) from Ilha Grande Bay, state of Rio de Janeiro. Revista Brasileira de Botânica 29: 27-37.

Taylor, W. 1960. Marine algae of the Eastern Tropical and Subtropical coast of the Americans. Michigan, The University of Michigan Press.

Underwood, A. 2000. Experimental ecology of rocky intertidal habitats: what are we learning? Journal of Experimental Marine Biology and Ecology 250: 51-76.

Underwood, A; Chapman M. \& Connell S. 2000. Observations in ecology: You can't make progress on processes without understanding the patterns. Journal of Experimental Marine Biology and Ecology 250: $97-115$.

Vreeland, V. \& Epstein L. 1996. Analysis of plant-substratum adhesives. Pp. 95-116. In: Linskens \& Jackson, J. (Eds.). Modern Methods of Plant Analysis. Plant Cell Wall Analysis. v. 17. Berlin, Springer-Verlag.

Wahl, M. 1989. Marine epibiosis: I. Fouling and antifouling: some basic aspects. Marine Ecology Progress Series 58: 175-189.

Wernberg, T. \& Connell S. 2008. Physical disturbance and subtidal habitat structure on open coasts: effects of wave exposure, extent and intensity. Journal of Sea Research 59: 237-248.

Wynne, M. 2011. A checklist of benthic marine algae of the tropical and subtropical western Atlantic: third revision. Beiheft Nova Hedwigia. 140: $1-166$.

Zayas, C.; Ocaña, F.; Suárez, A. \& La O, J. 2006. Variación estacional del epifitismo del fitobentos marino de Playa Guardalavaca, Cuba. Revista de Investigaciones Marinas 27: 95-101. 